\title{
Roflumilast reduziert Exazerbationen und die Anzahl der Krankenhauseinweisungen
}

\author{
Eine schwere chronisch-obstruktive Lungenerkrankung (COPD) \\ geht mit periodischen Exazerbationen der respiratorischen \\ Symptome einher, die oft eine aggressive Behandlung \\ erfordern. F. J. Martinez et al. haben im Rahmen einer großen \\ kontrollierten Studie überprüft, ob Roflumilast das Risiko einer \\ Verschlimmerung mindert. \\ Lancet 2015; 385: 857-866
}

Roflumilast ist ein oraler Phosphodiesterase-4-Inhibitor mit antiinflammatorischer Wirkung. Mit der REACT-Studie (Roflumilast and Exazerbations in patients receiving Appropriate Combination Therapy) wollten die Forscher nun herausfinden, ob Roflumilast auch bei Patienten mit schwerer COPD und einem hohen ExazerbationsRisiko wirkt. Bei diesen Patienten lässt sich die COPD nicht adäquat durch inhalierte Kombinationen von Kortikosteroiden mit langwirksamen 32 -Agonisten oder eine Dreifachkombinationstherapie von langwirksamen Muskarinantagonisten mit inhalierten Kortikosteroiden und langwirksamen $\beta 2$-Agonisten kontrollieren.

In die 1-jährige, doppelblinde, placebokontrollierte Studie der Phase 3 und 4 nahmen die Forscher in 203 Zentren in 21 Ländern Patienten mit schwerer COPD auf. In Frage kamen Patienten ab 40 Jahren mit einer Raucher-Vorgeschichte von mind. $20 \mathrm{~Pa}-$ ckungsjahren.Zudem sollten sie eine COPD mit schwerer Atmungseinschränkung, Symptome einer chronischen Bronchitis und mind. 2 Exazerbationen im vorangegangenen Jahr aufweisen. Die Forscher teilten die Patienten einer 1-mal täglichen Therapie mit 500 $\mu$ g Roflumilast oder Placebo zu. Alle Patienten erhielten eine fixe Kombination aus inhalativem Kortikosteroid und langwirksamem $\beta 2$-Agonisten. Zudem war bei Bedarf eine Therapie mit Tiotropiumbromid erlaubt. Primärer Endpunkt war die Exazerbationsrate der mäßigen bis schweren COPD pro Patient und Jahr. Die Auswertung erfolgte mittels Intention-to-treat-Analyse.

Von den 1945 geeigneten Patienten im mittleren Alter von 65 Jahren teilten die Untersucher 973 der Roflumilast- und 972 der Placebo-Gruppe zu. Wie die Poisson-
Regressionsanalyse ergab, lag die Rate der mäßigen bis schweren COPD-Exazerbationen in der Roflumilast- um 13,2\% niedriger als in der Placebogruppe. Entsprechend einer vordefinierten Sensitivitätsanalyse unter Anwendung einer negativen, binomialen Regression lag die Exazerbationsrate unter Verum um 14,2\% niedriger.

In $67 \%$ der Fälle traten unter Roflumilast und in 59\% unter Placebo Nebenwirkungen auf. Mit den Nebenwirkungen einhergehende Studienabbrüche waren in der Roflumilastgruppe (11\%) häufiger als unter Placebo (5\%). Die am häufigsten aufgeführten schweren Nebenwirkungen waren COPD-Exazerbationen (15 bzw. 19\%) und Pneumonie (4 bzw. 5\%). Im Vergleich zu Placebo lag die Anzahl schwerer Nebenwirkungen unter Roflumilast um 24,3\% und die Anzahl an Exazerbationen, die eine Krankenhauseinweisung erforderten, um 23,9\% niedriger. Unter Roflumilast traten 17 (1,8\%) und unter Placebo 18 (1,9\%) Todesfälle auf.

\section{Fazit}

Die Ergebnisse lassen nach Ansicht der Autoren darauf schließen, dass Roflumilast die Häufigkeit von Exazerbationen und Krankenhauseinweisungen bei Patienten mit schwerer COPD und chronischer Bronchitis reduziert, die trotz Gabe von inhalativen Kortikosteroiden und langwirkenden 32 -Agonisten selbst in Kombination mit Tiotropiumbromid, das Risiko häufiger und schwerer Exazerbationen aufweisen. Sie sind sich nach diesen positiven Ergebnissen mit Roflumilast sicher, dass weitere Erfolge bezüglich der zukünftigen Behandlungsmöglichkeiten von COPD zu erwarten sind.

Dr. Volker Kriegeskorte, Buchloe
Ausschreibung

\section{Forschungspreis für Seltene Erkrankungen}

Anlässlich des Tages der Seltenen Erkrankungen verleiht die Stiftung in Kooperation mit der Allianz Chronisch Seltener Erkrankungen (ACHSE) nun zum 9. Mal den mit $50000 €$ dotierten „Eva Luise Köhler Forschungspreis für Seltene Erkrankungen“. Die Preisverleihung erfolgt voraussichtlich am 28. Februar 2016 in Berlin. Ziel dieses Forschungspreises ist es, die Erforschung seltener Erkrankungen anhand beispielhafter Projekte voranzutreiben. Der Forschungspreis macht zudem Wissenschaft, Industrie und Gesellschaft darauf aufmerksam, dass die Forschung auf dem Gebiet der seltenen Erkrankungen zu den vordringlichen Aufgaben der Gesundheitspolitik gehören sollte.

Mit dem Eva-Luise-Köhler-Forschungspreis soll die Durchführung bzw. Anschubfinanzierung eines am Patientennutzen orientierten Forschungsprojektes im Bereich der seltenen Erkrankungen ermöglicht werden. Das Projekt soll einen innovativen Beitrag zur Verbesserung von Diagnostik, Prävention, Therapie oder zur Ursachenforschung bei seltenen Erkrankungen leisten und kurz-, mittel- oder langfristig zur Verbesserung der Lebensqualität von Patienten beitragen. Besonders preiswürdig sind nachhaltige, krankheitsübergreifende und forschungsvernetzende Projekte. Die Ergebnisse der Projektförderung sollten möglichst in einem fachwissenschaftlichen Publikationsmedium und durch eine Präsentation auf einer wissenschaftlichen Tagung veröffentlicht werden, um auch in der Fachwelt das Profil der Erforschung seltener Erkrankungen zu stärken.

Ausgefüllte Bewerbungsformulare können eingereicht werden bis einschließlich 6. September 2015. Das Bewerbungsformular kann als Datei online bezogen werden unter www.achse-online.de. Interessierte Wissenschaftler richten ihre Bewerbungen bitte ausschließlich per EMail an: Forschung@achse-online.de

Nach einer Mitteilung der ACHSE e.V., Berlin 